\section{Biosimilar zur Prophylaxe von Neutropenie}

\begin{abstract}
Die EORTC-Leitlinie empfiehlt zur Prävention chemotherapieinduzierter Neutropenien Biosimilars von Filgrastim ebenso wie das Referenzprodukt. Bei Patientinnen mit Mammakarzinom, die eine (neo)adjuvante myelosuppressive Chemotherapie erhielten, wurden beide Optionen verglichen.
\end{abstract}

\begin{abstract}
A n der randomisierten Phase-III-Studie nahmen 218 Patientinnen teil, die eine (neo)adjuvante Chemotherapie mit $75 \mathrm{mg} / \mathrm{m}^{2}$ Docetaxel, $50 \mathrm{mg} / \mathrm{m}^{2}$ Doxorubicin und $500 \mathrm{mg} / \mathrm{m}^{2}$ Cyclophosphamid (TAC) erhielten. Filgrastim (das Filgrastim-Biosimilar EP2006 oder das Referenzprodukt Neupogen ${ }^{\circledast}$ ) wurde in einer Dosierung von $5 \mu \mathrm{g} / \mathrm{kg}$ pro Tag über die 6 Chemotherapiezyklen verabreicht. Dabei wurde im Verhältnis 1:1:1:1 auf vier Arme randomisiert: Zwei Therapiearme erhielten jeweils nur ein Produkt, das Biosimilar oder das Referenzprodukt (nicht alternierende Gruppen), in den anderen beiden Armen (alternierende Gruppen) wurde das Produkt während eines jeden Zyklus gewechselt
\end{abstract}

(Biosimilar, anschließend Referenzprodukt oder umgekehrt).

Die Ergebnisse bestätigten die Nichtunterlegenheit des Biosimilars: Die Dauer schwerer Neutropenien während des ersten Zyklus betrug unter Biosimilar ( $n=101) 1,17 \pm 1,11$ Tage, unter dem Referenzprodukt $(\mathrm{n}=103) 1,20 \pm 1,02$ Tage (untere Grenze des 97,5\%-Konfidenzintervalls -0,26 Tage - oberhalb des prädefinierten Limits von -1 Tag). Bezüglich anderer Effizienzparameter waren keine bedeutsamen Unterschiede zu verzeichnen: Hospitalisierung wegen febriler Neutropenie, Inzidenz von Infektionen, Tiefe und Zeitpunkt des Nadirs der Neutrophilenzahl, Zeit bis zur Erholung der Neutrophilen während des ersten Zyklus bzw. durch alle Zyklen. Die Zahl der Patientinnen, die eine febrile Neutropenie entwickelten, war im Biosimilar-Arm zwar höher (4,7 vs. 1,9\%) - der Unterschied rangierte den Wissenschaftlern zufolge jedoch im erwarteten Rahmen der Variabilität. Auch Muster und Frequenz von Nebenwirkungen glichen sich in den vier Therapiearmen.

Fazit: In der Studie erwiesen sich bei Patientinnen mit Mammakarzinom das Biosimilar Filgrastim EP2006 und sein Referenzprodukt in der Prophylaxe schwerer Neutropenie bei myelosuppressiver Chemotherapie einander ebenbürtig. Dies, so Kimberly L. Blackwell und Kollegen, erleichtere vermutlich mehr Patientinnen den Zugang zu einer Therapie mit Filgrastim. Kathrin von Kieseritzky

Blackwell K et al. Comparison of EP2006, a Filgrastim Biosimilar, to the Reference: a Phase III, Randomized, Double-Blind Clinical Study in the Prevention of Severe Neutropenia in Patients with Breast Cancer Receiving Myelosuppressive Chemotherapy. Ann Oncol. 2015 ;26(9):1948-53.

\section{Hand-Fuß-Syndrom: Wie vorbeugen?}

\begin{abstract}
Unter einer Therapie mit Capecitabin, pegyliertem liposomalem Doxorubicin oder manchen Tyrosinkinaseinhibitoren kommt es nicht selten zu einem HandFuß-Syndrom (HFS). Eine Standardprophylaxe gibt es bislang nicht.
\end{abstract}

\begin{abstract}
$\mathrm{n}$ einer randomisierten Phase-III-Studie wurde die Wirksamkeit von Mapisal im Vergleich zu einer 10\%igen UreaCreme bei 152 Patienten mit gastrointestinalen Tumoren oder Brustkrebs getestet, die Capecitabin erhielten. Mapisal
\end{abstract}

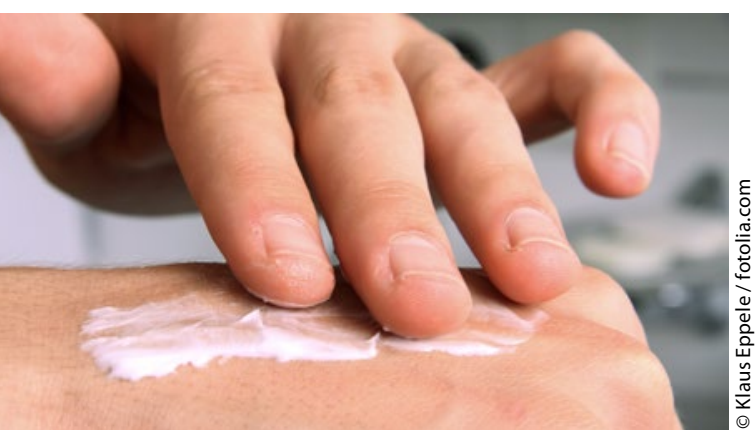

Dreimal täglich Urea-Creme kann zur Prophylaxe des HFS empfohlen werden. enthält verschiedene Antioxidanzien und Öl-Extrakte. Im Verhältnis 1:1 randomisiert sollten die Patienten entweder Mapisal oder Urea-Creme jeweils 3-mal täglich sowie nach jedem Händewaschen für insgesamt 6 Wochen anwenden. Weitere Hautpflegeprodukte durften in dieser Zeit an Händen und Füßen nicht angewendet werden.

39,5\% der Patienten in der MapisalGruppe entwickelten ein HFS (Grad 1: 66,7\%; Grad 2: 26,7\%; Grad 3: 6,7\%). Unter der Urea-Creme waren es $22,4 \%$ (Grad 1: 52,9\%; Grad 2: 41,2\%; Grad 3: 5,9\%). Unter der Mapisal-Prophylaxe war der Anteil der Patienten mit HFS also signifikant höher (stratifizierte Odds Ratio 2,37; $\mathrm{p}=0,02)$. Die Zeit bis zur Entwicklung eines HFS > Grad 1 war in beiden Gruppen vergleichbar. Jedoch dauerte es in der Urea-Creme-Gruppe signifikant länger, bis ein HFS jedweden Grades auftrat $(\mathrm{p}=0,03)$. Die angewendete Capecitabin-Dosis und die Zeit, innerhalb derer die Studienmedikation korrekt angewendet wurde, waren in den beiden Therapiearmen identisch, ebenso andere Nebenwirkungen. Zwei Nebenwirkungen wurden Mapisal zugeschrieben: Ein Patient entwickelte ein Erythema multiforme, ein Patient makulopapulösen Rash, beides jeweils vom Grad 1. Die hautbezogene Lebensqualität war am Ende der Studie in der Mapisal-Gruppe signifikant schlechter als in der Urea-Creme-Gruppe.

Fazit: 10\%ige Urea-Creme erwies sich in der Prävention des HFS bei Patienten, die eine Chemotherapie mit Capecitabin erhielten, gegenüber Mapisal als überlegen - nach Ansicht der Wissenschaftler ein Anlass, Urea-Creme derzeit als Standardprophylaxe für künftige Studien zu empfehlen.

Kathrin von Kieseritzky

Hofheinz RD et al. Mapisal Versus Urea Cream as Prophylaxis for Capecitabine-Associated HandFoot Syndrome: A Randomized Phase III Trial of the AIO Quality of Life Working Group. J Clin Oncol. 2015;33(22):2444-49. 